\title{
HBV ccCDNA and Its Potential as a Therapeutic Target
}

\author{
Anjing Zhu, Xinzhong Liao, Shuang Li, Hang Zhao, Limin Chen, Min Xu* and Xiaoqiong Duan* \\ Institute of Blood Transfusion, Chinese Academy of Medical Sciences and Peking Union Medical College, Chengdu, China
}

\begin{abstract}
Chronic hepatitis B virus infection continues to be a major health burden worldwide. It can cause various degrees of liver damage and is strongly associated with the development of liver cirrhosis and hepatocellular carcinoma. Covalently closed circular DNA in the nucleus of infected cells cannot be disabled by present therapies which may lead to HBV persistence and relapse. In this review, we summarized the current knowledge on hepatitis B virus covalently closed circular DNA and its potential role as a therapeutic target.

Citation of this article: Zhu A, Liao X, Li S, Zhao H, Chen L, $\mathrm{Xu} \mathrm{M}$, et al. HBV cccDNA and its potential as a therapeutic target. J Clin Transl Hepatol 2019;7(3):258-262. doi: 10.14218/JCTH.2018.00054.
\end{abstract}

\section{Hepatitis B virus (HBV) life cycle and therapies}

HBV was discovered by Blumberg and colleagues ${ }^{1}$ in 1965 . Today, there are about 257 million people chronically infected with HBV, which causes 650000 deaths worldwide every year. According to World Health Organization reports, HBV caused 887000 deaths, mostly from complex diseases (including cirrhosis and hepatocellular carcinoma) in 2015. HBV infection brings a huge burden to people and even society.

The HBV genome is a circular, partially double-stranded DNA, which is enveloped by an outer lipoprotein with an inner nucleocapsid core. HBV DNA is only $3.2 \mathrm{~kb}$ in length and contains four overlapping open reading frames, known as $S$, $C, P$ and $X$. The four open reading frames code for 7 viral proteins: pre-S1, pre-S2, S, C, pre-C, X protein (commonly known as HBX), and HBV polymerase. ${ }^{2-4}$ Once infected, HBV enters host hepatocytes by binding to the sodium taurocholate cotransporting polypeptide NTCP and being endocytosed. ${ }^{5}$ After entry, the virus releases its DNA-containing nucleocapsid into the cytoplasm, which is then transported to the nucleus. In the nucleus, the viral DNA is converted from its relaxed circular form to closed covalent circular DNA (cccDNA). ${ }^{6-9}$ The cccDNA is transcribed into HBV pregenomic RNA (pgRNA) and several subgenomic RNAs.6,10

Keywords: Hepatitis B virus (HBV); Covalently closed circular DNA (cccDNA); Therapeutic target.

Abbreviations: ccCDNA, covalently closed circular DNA; CRISPR/Cas, clustered regularly interspaced short palindromic repeats/CRISPR associated; HBV, chronic hepatitis B virus; NA, nucleotide analogue; pgRNA, pregenomic RNA; TALEN, transcription activator-like effector nuclease; ZFN, zinc finger nuclease.

Received: 26 September 2018; Revised: 2 April 2019; Accepted: 10 July 2019 *Correspondence to: Min Xu and Xiaoqiong Duan, Institute of Blood Transfusion, Chinese Academy of Medical Sciences and Peking Union Medical College, Chengdu 610052, China. Tel: +86-135-4080-7307, E-mail: xumin@ibt.pumc.edu.cn (MX) or xiaoqiongduan@ibt.pumc.edu.cn (XD)
Integration of the HBV DNA into the host genome begins immediately after infection of hepatocytes. ${ }^{11}$ After transcription and translation, pgRNA is packaged and assembled in the nucleocapsid, where it is transcribed to relaxed circular DNA. Finally, the nucleocapsid is enveloped and the virion is secreted from the hepatocyte. ${ }^{12,13}$

The current standard therapy for HBV infection includes PEGylated interferon $\alpha$ and nucleotide analogues (NAs). For example, a recent study showed that interferon $\alpha$ induces a long-term and sustainable suppression of cccDNA transcription, possibly by altering epigenetic modification of cccDNA minichromosomes. ${ }^{13}$ The inhibition of HBV replication by NAs is mediated by targeting the viral RNA-dependent DNA polymerase which catalyzes the reverse transcription of pgRNA to mature viral DNA. Five NAs have been approved for clinical use since 1998, including lamivudine, entecavir, telbivudine, adefovir dipivoxil and tenofovir. ${ }^{14}$ Although NAs have been considered as first-line therapy for the treatment of chronic HBV infection due to their high efficiency, ${ }^{13}$ they have failed to cure HBV in most cases because they cannot clear cccDNA. ${ }^{15}$ As such, virus rebound occurs following the termination of NAs treatment. Obviously, disabling the cccDNA is key in terms of curing HBV infection. ${ }^{16}$

\section{HBV ccCDNA: Formation and modification}

cccDNA is stable and acts as a virus transcription template. There are 3-50 copies of cccDNA per infected cell, and the number of copies decreases when the host cell divides. Thus, recycling of the new relaxed circular form to the nucleus occurs in order to maintain the relatively stable cccDNA copy number. ${ }^{17}$ There are three main steps in conversion of the relaxed circular form to cccDNA: (1) unlocking of the protein, which is covalently linked to the $5^{\prime}$ end of the (-)-DNA; (2) removal of the $5^{\prime}$ end of the (+)-strand consisting of an RNA oligonucleotide; and (3) covalently ligation of both strands. ${ }^{18,19}$ Recent studies have shown that the host DNA damage response is involved in the formation of cccDNA, but the exact mechanism remains to be clarified. ${ }^{20}$ Epigenetic modifications, like histone acetylation and DNA methylation, play an important role in the transcriptional activity of cccDNA. ${ }^{21-24}$ Six CpG islands have been reported in the HBV genome. The $\mathrm{CpG}$ islands distribute differently in HBV genotypes, overlapping some functional genes. Three conventional CpG islands (I, II, III) are potential targets for HBV DNA methylation (Fig. 1). ${ }^{25}$

\section{Novel strategies for eradicating HBV cccDNA}

HBV cure depends on disabling of the HBV cccDNA, which is very difficult because cccDNA resides in the nucleus as an 


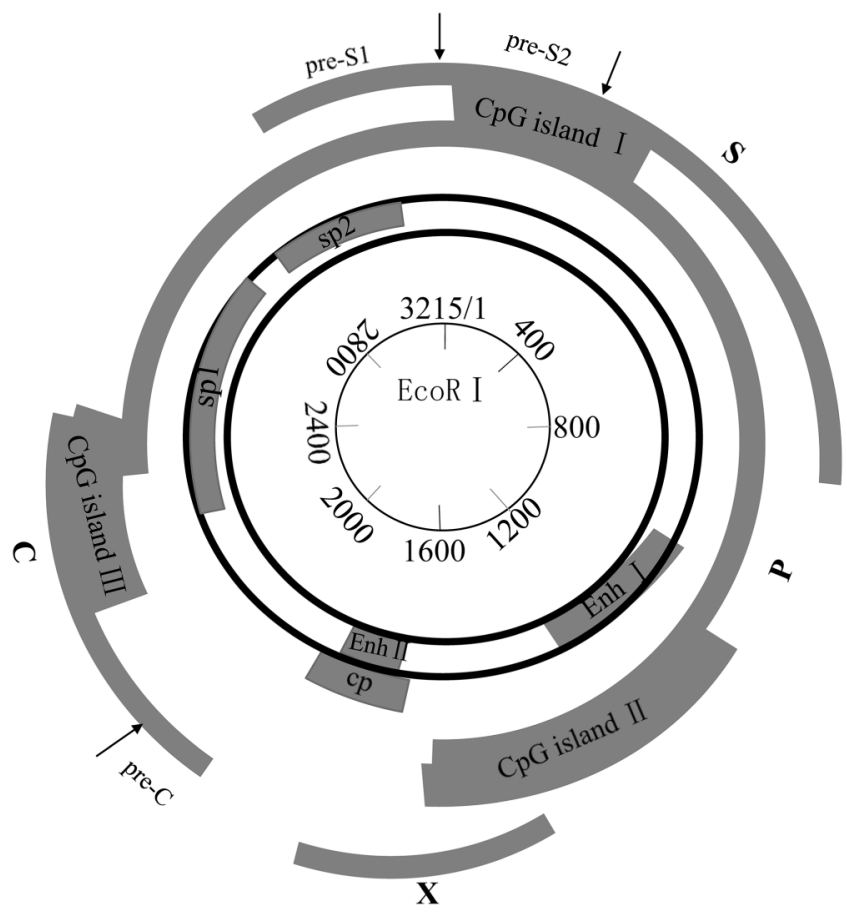

Fig. 1. Distribution diagram of CpG islands. Island I overlaps the start site of the $\mathrm{S}$ gene; island II contains enhance I and the $\mathrm{X}$ gene promoter, close to the core gene promoter and enhance II; island III overlaps the Sp1 promoter and the start codon of the P gene.

episomal plasmid-like molecule to produce progeny virus. ${ }^{15,26}$ Several gene therapy strategies have been proposed to disable HBV ccCDNA.

\section{Silencing cccDNA expression by gene editing techniques}

Several genomic editing technologies, including zinc finger nucleases (ZFNs), transcription activator-like effector nucleases (TALENs) and the clustered regularly interspaced short palindromic repeats/CRISPR associated (CRISPR/Cas) system, have been used to disrupt HBV ccCDNA.

ZFNs, a custom DNA endonuclease, are used to create a DNA double-strand break in a specific target site and repair by creating sequence alterations at the cleavage sites. ${ }^{27-29}$ Through a proof-of-concept experiment, scientists found that anti-HBV ZFNs (cognate 6L and 6R ZFN pair) disrupted $36 \%$ of plasmid-derived viral sequences in a cell culture model. Weber et al. ${ }^{30}$ designed three ZFNs targeting HBV polymerase, gene $X$ and core, and delivered them into the HepAD38 cells by self-complementary adeno-associated viral vectors, respectively. They found these HBV-targeted ZFNs produced a sustained suppression of HBV levels over the course of the experiment. ${ }^{31}$

TALENs are similar to ZFNs. However, the DNA-binding domain of TALENs is highly repeated and derived from transcription activator-like III effectors, which are proteins secreted by Xanthomonas bacteria. ${ }^{32,33}$ The efficiency of TALENs in reducing HBV productions in cell culture was first described by Bloom et al. ${ }^{34}$ in 2013 . The investigators found that targeting the $\mathrm{S}$ and $\mathrm{C}$ regions of cccDNA led to $35 \%$ decrease in cccDNA molecules in HepG2 cell lines. Moreover, gene editing can be used in combination with gene-silencing technologies for antiHBV therapy. Using TALEN-mediated homology directed recombination to introduce artificial primary miRNAs into HBV genome could boost the antiviral efficacy of TALENs. ${ }^{35}$

The CRISPR/Cas system is the adaptive immunity of bacteria and archaea, and acts against invading foreign DNA via RNA-guided DNA cleavage. ${ }^{36-40}$ The CRISPR/Cas9 system is a newly developed programmable genome-editing tool and allows for sequence-specific cleavage of DNA. Compared to other genome-editing tools, the advantage of the CRIPSR/ Cas9 system lies in its simplicity and flexibility in design. Several studies have demonstrated that the CRISPR/Cas9 system can efficiently destroy HBV cCCDNA. ${ }^{41-45}$ Using the CRISPR/Cas9 system, scientists have completely excised a full-length 3175bp integrated HBV DNA from the host genome and disrupted the HBV cccDNA in a stable HBV cell line. ${ }^{44,45}$ Therefore, gene editing seems highly promising for disabling HBV cccDNA. ${ }^{29,34}$

These three genome-editing technologies work similarly by targeting and modifying DNA sequences using engineered nucleases, thereby inducing a targeted DNA double-strand break that stimulates the cellular DNA repair mechanisms. However, the engineered nucleases, recognition area, and the molecular mechanism are different. ${ }^{46}$ Engineered ZFNs and TALENs are composed of a DNA binding domain and a Fok I nuclease motif. The CRISPR-Cas9 genome editing system consists of two components: a "guide" RNA and a nonspecific CRISPR-associated endonuclease (the Cas9). The recognition areas of the ZFNs, TALENs, CRISPR/Cas system are zincfinger proteins, NLS, and sgRNA, respectively. ZFNs and TALENs use the Fok I enzyme to cleave target DNA, while CRISPR/Cas uses the Cas protein. The target sizes among the three genome-editing tools are also different, that is (9-12bp)*2 for ZFNs and TALENs, 20bp+ NGG for CRISPR/ Cas. ${ }^{29,46,47}$

There are some limitations for these technologies. First, specificity can be altered by context-dependent effects caused by interactions among neighboring zinc fingers of the DNA binding domain' Second, the large size of TALENs makes it difficult to deliver. Finally, the CRISPR/Cas9 system faces several challenges like in vivo delivery efficiency and off-target cleavage. ${ }^{48,49}$ Cutting integrated HBV genomes by CRISPR/Cas9 also raises serious concerns, because this manipulation can cause genome instability. Compared to ZFNs and TALENs, the two advantages of the CRISPR/Cas9 system have contributed to the advancement of the new technology and generated widespread interest, according to its simplicity and flexibility. Thus, we believe that the CRISPR/ Cas9 system is very promising for curing chronic HBV once several challenges are solved. ${ }^{50}$

\section{Silencing ccCDNA transcription by epigenetic modifications}

HBV cccDNA exists in the nucleus as multiple copies of nucleosome-decorated minichromosomes, which indicates that epigenetic modifications may influence HBV replication and persistent infection. ${ }^{51}$ It has been shown that DNA methylation and histone acetylation are required for cccDNA formation. ${ }^{22}$ Therefore, regulation of DNA methylation or histone acetylation is a potential method to reduce cccDNA. ${ }^{21,52-55}$ Interferon $\alpha$ treatment has been adopted to silence cccDNA through epigenetic modification. It has been 
found that interferon $\alpha$ inhibits HBV replication by regulating the epigenetic modification of HBV cccDNA. Interferon $\alpha$ represses the transcription of HBV ccCDNA through recruitment of the histone deacetylases HDAC1 and hSirt1 and decreasing the acetylation of cccDNA-bound histones. ${ }^{56}$

Hyun et al. ${ }^{24}$ found that short hairpin RNA induced HBV cccDNA methylation to inhibit its transcription in human hepatoma cells. HBV cccDNA transcription was regulated by $\mathrm{CpG}$ methylation during chronic HBV infection. ${ }^{21} \mathrm{CpG}$ island II methylation has been shown to significantly decrease ccCDNA transcription and subsequent viral core DNA replication, while CpG island III methylation has been shown to be associated with low serum HBsAg titers (Fig. 1). ${ }^{21,53,54,57-59}$ Interestingly, $\mathrm{HBx}$ can reduce chromatin-mediated transcriptional repression of HBV ccCDNA caused by SETDB1 histone methyltransferase and therefore allow the establishment of active chromatin. ${ }^{60}$ Recently, it has also been reported that HB core carboxyl-terminal domain arginine residues reduced acetylation of cccDNA-bound histones and thus reduced the interaction of $\mathrm{HBC}$ with cccDNA. ${ }^{61}$ Two enzymes have been found to regulate the methylation. ${ }^{62,63}$ Protein arginine methyltransferase 5 can regulate symmetric dimethylation of arginine 3 on histone 4 of cccDNA. ${ }^{62}$ The silent mating type information regulation 2 homolog 3 SIRT3 restricts HBV transcription and replication via epigenetic regulation of CCCDNA, involving SUV39H1 and SETD1A histone methyltransferases. ${ }^{63}$

HBV replication is also regulated by the acetylation status of HBV CCCDNA-bound histone 3 and histone $4 .^{22}$ The Np95/ ICBP90-like RING finger protein NIRF, a novel E3 ubiquitin ligase, has been found to inhibit HBV DNA replication and hepatitis $B$ e antigen secretion in HepG2 cells through reducing HBV ccCDNA-bound histone $3 .{ }^{64}$ Curcumin can inhibit HBV replication via down-regulation of ccCDNA-bound histone acetylation. ${ }^{65}$ Retinoid $X$ receptor $\alpha$ was also reported to be able to regulate the replication of $\mathrm{HBV}$ and modulate the HBV ccCDNA epigenetically. ${ }^{66}$ In addition, basal core promoter mutations were found to inhibit viral replication through modulating the acetylation and deacetylation of cccDNA-bound histones, while preCore mutations have been shown to have no effect on viral replication. ${ }^{67}$ These collective findings indicate that regulation of the DNA methylation and histone acetylation may inactivate cccDNA transcription and thus inhibit HBV replication.

Disabling cccDNA is the key to curing hepatitis B. Studies have shown the great potential of gene editing technologies for disrupting the cccDNA in cultured cells and in hydrodynamically-injected mice. However, in the case of HBV infection, there are some disadvantages of using gene editing technologies. First, HBV DNA often integrates into the host genome and undesirable gene cleavage may occur. Second, off-target effects has been observed in some studies. Finally, the means by how to deliver in vivo remains a challenge for therapeutic application of these technologies. The epigenetic modifications mentioned above are related to the replication and transcription of HBV ccCDNA. While they can regulate the HBV CCCDNA, they cannot eliminate the HBV ccCDNA.

\section{Conclusions}

In conclusion, disabling or complete inactivation of cccDNA is the desired end-point of HBV treatment. However, the most urgent issues should be addressed before using gene editing technologies for HBV treatment include solving the off-target problem and finding ways to deliver in vivo. What is more, for a long time, lack of robust, reliable and quantifiable HBV cccDNA models has delayed the development of cccDNA therapies. Recently, Yuan et al. ${ }^{68}$ established a cell line through integrating 2-60 copies of the monomeric HBV genome into HepG2-derived cell lines, where the cccDNA could be produced and detected with specific primers. The establishment of a cell line will provide a proper model for evaluation of drugs or therapies targeting on cccDNA. Though there are challenges to overcome for both gene editing- and epigenetic modification-based strategies and it there remains a long road from basic research to clinical application, it is very likely that we will conquer HBV similar to the hepatitis $C$ virus cure in the future.

\section{Conflict of interest}

The authors have no conflict of interests related to this publication.

\section{Author contributions}

Collected the data and wrote the manuscript ( $A Z, X D)$, revised the manuscript and answered the reviewers questions $(A Z, M X)$, directed and wrote the manuscript with comments (LC), helped to edit the manuscript ( $\mathrm{XL}, \mathrm{SL}, \mathrm{HZ}$ ). All authors have seen and approved the content of this manuscript.

\section{References}

[1] Blumberg BS, Gerstley BJ, Hungerford DA, London WT, Sutnick AI. A serum antigen (Australia antigen) in Down's syndrome, leukemia, and hepatitis. Ann Intern Med 1967:66:924-931. doi: 10.7326/0003-4819-66-5-924.

[2] Tiollais P, Pourcel C, Dejean A. The hepatitis B virus. Nature 1985;317:489495. doi: 10.1038/317489a0.

[3] Arzumanyan A, Reis HM, Feitelson MA. Pathogenic mechanisms in HBV- and HCV-associated hepatocellular carcinoma. Nat Rev Cancer 2013;13:123135. doi: $10.1038 /$ nrc3449.

[4] Doo EC, Ghany MG. Hepatitis B virology for clinicians. Clin Liver Dis 2010;14: 397-408. doi: 10.1016/j.cld.2010.05.001.

[5] Li W. The hepatitis B virus receptor. Annu Rev Cell Dev Biol 2015;31:125147. doi: 10.1146/annurev-cellbio-100814-125241.

[6] Seeger C, Mason WS. Hepatitis B virus biology. Microbiol Mol Biol Rev 2000; 64:51-68. doi: 10.1128/mmbr.64.1.51-68.2000.

[7] Bock CT, Schranz P, Schröder CH, Zentgraf $H$. Hepatitis B virus genome is organized into nucleosomes in the nucleus of the infected cell. Virus Genes 1994;8:215-229. doi: 10.1007/bf01703079.

[8] Newbold JE, Xin H, Tencza M, Sherman G, Dean J, Bowden S, et al. The covalently closed duplex form of the hepadnavirus genome exists in situ as a heterogeneous population of viral minichromosomes. J Virol 1995;69: 3350-3357.

[9] Zoulim F. New insight on hepatitis B virus persistence from the study of intrahepatic viral cccDNA. J Hepatol 2005;42:302-308. doi: 10.1016/j.jhep. 2004.12.015

[10] Block TM, Guo H, Guo JT. Molecular virology of hepatitis B virus for clinicians. Clin Liver Dis 2007;11:685-706. doi: 10.1016/j.cld.2007.08.002.

[11] Tu T, Budzinska MA, Vondran FWR, Shackel NA, Urban S. Hepatitis B Virus DNA Integration Occurs Early in the Viral Life Cycle in an In Vitro Infection Model via Sodium Taurocholate Cotransporting Polypeptide-Dependent Uptake of Enveloped Virus Particles. J Virol 2018;92:e02007-17. doi: 10. 1128/JVI.02007-17.

[12] Huovila AP, Eder AM, Fuller SD. Hepatitis B surface antigen assembles in a post-ER, pre-Golgi compartment. J Cell Biol 1992;118:1305-1320. doi: 10. 1083/jcb.118.6.1305.

[13] Tang LSY, Covert E, Wilson E, Kottilil S. Chronic hepatitis B infection: A review. JAMA 2018;319:1802-1813. doi: 10.1001/jama.2018.3795.

[14] Liaw YF, Sung J], Chow WC, Farrell G, Lee CZ, Yuen H, et al. Lamivudine for patients with chronic hepatitis B and advanced liver disease. N Engl J Med 2004;351:1521-1531. doi: 10.1056/NEJMoa033364.

[15] Zhou T, Guo H, Guo JT, Cuconati A, Mehta A, Block TM. Hepatitis B virus e antigen production is dependent upon covalently closed circular (ccc) DNA in 
HepAD38 cell cultures and may serve as a cccDNA surrogate in antiviral screening assays. Antiviral Res 2006;72:116-124. doi: 10.1016/j.antiviral. 2006.05.006.

[16] Seeger C, Mason WS. Molecular biology of hepatitis B virus infection. Virology 2015;479-480:672-686. doi: 10.1016/j.virol.2015.02.031.

[17] Ji M, Hu K. Recent advances in the study of hepatitis $B$ virus covalently closed circular DNA. Virol Sin 2017;32:454-464. doi: 10.1007/s12250-017-4009-4.

[18] Qi Y, Gao Z, Xu G, Peng B, Liu C, Yan H, et al. DNA polymerase $\kappa$ is a key cellular factor for the formation of covalently closed circular DNA of hepatitis B virus. PLoS Pathog 2016;12:e1005893. doi: 10.1371/journal.ppat. 1005893.

[19] Königer C, Wingert I, Marsmann M, Rösler C, Beck J, Nassal M. Involvement of the host DNA-repair enzyme TDP2 in formation of the covalently closed circular DNA persistence reservoir of hepatitis B viruses. Proc Natl Acad Sci U S A 2014;111:E4244-E4253. doi: 10.1073/pnas.1409986111.

[20] Schreiner S, Nassal M. A role for the host DNA damage response in hepatitis B virus cccDNA formation-and beyond? Viruses 2017;9:E125. doi: 10 . 3390/v9050125.

[21] Kim JW, Lee SH, Park YS, Hwang JH, Jeong SH, Kim N, et al. Replicative activity of hepatitis $B$ virus is negatively associated with methylation of covalently closed circular DNA in advanced hepatitis B virus infection. Intervirology 2011;54:316-325. doi: 10.1159/000321450.

[22] Pollicino T, Belloni L, Raffa G, Pediconi N, Squadrito G, Raimondo G, et al. Hepatitis $B$ virus replication is regulated by the acetylation status of hepatitis $B$ virus cccDNA-bound H3 and H4 histones. Gastroenterology 2006;130: 823-837. doi: 10.1053/j.gastro.2006.01.001.

[23] Liu F, Campagna M, Qi Y, Zhao X, Guo F, Xu C, et al. Alpha-interferon suppresses hepadnavirus transcription by altering epigenetic modification of ccCDNA minichromosomes. PLoS Pathog 2013;9:e1003613. doi: 10. 1371/journal.ppat.1003613.

[24] Park HK, Min BY, Kim NY, Jang ES, Shin CM, Park YS, et al. Short hairpin RNA induces methylation of hepatitis $B$ virus covalently closed circular DNA in human hepatoma cells. Biochem Biophys Res Commun 2013;436:152155. doi: $10.1016 / j$.bbrc.2013.04.108.

[25] Zhang Y, Li C, Zhang Y, Zhu H, Kang Y, Liu H, et al. Comparative analysis of CpG islands among HBV genotypes. PLoS One 2013;8:e56711. doi: 10. 1371 /journal.pone.0056711.

[26] Werle-Lapostolle B, Bowden S, Locarnini S, Wursthorn K, Petersen J, Lau G, et al. Persistence of cccDNA during the natural history of chronic hepatitis B and decline during adefovir dipivoxil therapy. Gastroenterology $2004 ; 126$ : 1750-1758. doi: 10.1053/j.gastro.2004.03.018.

[27] Cathomen T, Keith Joung J. Zinc-finger nucleases: The next generation emerges. Mol Ther 2008;16:1200-1207. doi: 10.1038/mt.2008.114.

[28] Kim YG, Cha J, Chandrasegaran S. Hybrid restriction enzymes: zinc finger fusions to Fok I cleavage domain. Proc Natl Acad Sci U S A 1996;93:11561160. doi: 10.1073/pnas.93.3.1156.

[29] Cradick TJ, Keck K, Bradshaw S, Jamieson AC, McCaffrey AP. Zinc-finger nucleases as a novel therapeutic strategy for targeting hepatitis $B$ virus DNAs. Mol Ther 2010;18:947-954. doi: 10.1038/mt.2010.20.

[30] Weber ND, Stone D, Sedlak RH, De Silva Feelixge HS, Roychoudhury $P$, Schiffer JT, et al. AAV-mediated delivery of zinc finger nucleases targeting hepatitis B virus inhibits active replication. PLoS One 2014;9:e97579. doi: 10.1371/journal.pone.0097579.

[31] Bloom K, Maepa MB, Ely A, Arbuthnot P. Gene therapy for chronic HBV-Can we eliminate cccDNA? Genes (Basel) 2018;9:E207. doi: 10. 3390/genes9040207.

[32] Maeder ML, Thibodeau-Beganny S, Osiak A, Wright DA, Anthony RM, Eichtinger $M$, et al. Rapid "open-source" engineering of customized zinc-finger nucleases for highly efficient gene modification. Mol Cell 2008;31:294-301. doi: 10.1016/j.molcel.2008.06.016.

[33] Boch J, Bonas U. Xanthomonas AvrBs3 family-type III effectors: discovery and function. Annu Rev Phytopathol 2010;48:419-436. doi: 10. 1146/annurev-phyto-080508-081936.

[34] Bloom K, Ely A, Mussolino C, Cathomen T, Arbuthnot P. Inactivation of hepatitis $B$ virus replication in cultured cells and in vivo with engineered transcription activator-like effector nucleases. Mol Ther 2013;21:1889-1897. doi: 10.1038/mt.2013.170.

[35] Dreyer T, Nicholson S, Ely A, Arbuthnot P, Bloom K. Improved antiviral efficacy using TALEN-mediated homology directed recombination to introduce artificial primary miRNAs into DNA of hepatitis B virus. Biochem Biophys Res Commun 2016;478:1563-1568. doi: 10.1016/j.bbrc.2016.08.152.

[36] Wiedenheft B, Sternberg SH, Doudna JA. RNA-guided genetic silencing systems in bacteria and archaea. Nature 2012;482:331-338. doi: 10. 1038 /nature10886.

[37] Shah SA, Garrett RA. CRISPR/Cas and Cmr modules, mobility and evolution of adaptive immune systems. Res Microbiol 2011;162:27-38. doi: 10. 1016/j.resmic.2010.09.001.

[38] Jore MM, Brouns S], van der Oost J. RNA in defense: CRISPRs protect prokaryotes against mobile genetic elements. Cold Spring Harb Perspect Biol 2012;4:a003657. doi: 10.1101/cshperspect.a003657.
[39] Garneau JE, Dupuis MÈ, Villion M, Romero DA, Barrangou R, Boyaval P, et al. The CRISPR/Cas bacterial immune system cleaves bacteriophage and plasmid DNA. Nature 2010;468:67-71. doi: 10.1038/nature09523.

[40] Manica A, Zebec Z, Teichmann D, Schleper C. In vivo activity of CRISPRmediated virus defence in a hyperthermophilic archaeon. Mol Microbiol 2011;80:481-491. doi: 10.1111/j.1365-2958.2011.07586.x.

[41] Seeger C, Sohn JA. Complete Spectrum of CRISPR/Cas9-induced Mutations on HBV cccDNA. Mol Ther 2016;24:1258-1266. doi: 10.1038/mt.2016.94.

[42] Ramanan V, Shlomai A, Cox DB, Schwartz RE, Michailidis E, Bhatta A, et al. CRISPR/Cas9 cleavage of viral DNA efficiently suppresses hepatitis $B$ virus. Sci Rep 2015;5:10833. doi: 10.1038/srep10833.

[43] Karimova M, Beschorner N, Dammermann W, Chemnitz J, Indenbirken $D$, Bockmann JH, et al. CRISPR/Cas9 nickase-mediated disruption of hepatitis $B$ virus open reading frame $S$ and X. Sci Rep 2015;5:13734. doi: 10. $1038 /$ srep 13734.

[44] Liu X, Hao R, Chen S, Guo D, Chen Y. Inhibition of hepatitis B virus by the CRISPR/Cas9 system via targeting the conserved regions of the viral genome. J Gen Virol 2015;96:2252-2261. doi: 10.1099/vir.0.000159.

[45] Li H, Sheng C, Wang S, Yang L, Liang Y, Huang $Y$, et al. Removal of integrated hepatitis B virus DNA using CRISPR-Cas9. Front Cell Infect Microbiol 2017;7: 91. doi: $10.3389 /$ fcimb.2017.00091.

[46] Gaj T, Gersbach CA, Barbas CF 3rd. ZFN, TALEN, and CRISPR/Cas-based methods for genome engineering. Trends Biotechnol 2013;31:397-405. doi: 10.1016/j.tibtech.2013.04.004.

[47] Holkers M, Maggio I, Liu J, Janssen JM, Miselli F, Mussolino C, et al. Differential integrity of TALE nuclease genes following adenoviral and lentiviral vector gene transfer into human cells. Nucleic Acids Res 2013;41:e63. doi: $10.1093 / \mathrm{nar} / \mathrm{gks} 1446$.

[48] Mussolino C, Morbitzer R, Lütge F, Dannemann N, Lahaye T, Cathomen T. A novel TALE nuclease scaffold enables high genome editing activity in combination with low toxicity. Nucleic Acids Res 2011;39:9283-9293. doi: 10. 1093/nar/gkr597.

[49] Tsai SQ, Zheng Z, Nguyen NT, Liebers M, Topkar VV, Thapar V, et al. GUIDEseq enables genome-wide profiling of off-target cleavage by CRISPR-Cas nucleases. Nat Biotechnol 2015;33:187-197. doi: 10.1038/nbt.3117.

[50] Yang HC, Chen PJ. The potential and challenges of CRISPR-Cas in eradication of hepatitis B virus covalently closed circular DNA. Virus Res 2018;244:304310. doi: 10.1016/j.virusres.2017.06.010.

[51] Levrero M, Pollicino T, Petersen J, Belloni L, Raimondo G, Dandri M. Control of cccDNA function in hepatitis B virus infection. J Hepatol 2009;51:581-592. doi: $10.1016 /$ j.jhep.2009.05.022.

[52] Miller RH, Robinson WS. Integrated hepatitis B virus DNA sequences specifying the major viral core polypeptide are methylated in $\mathrm{PLC} / \mathrm{PRF} / 5$ cells. Proc Natl Acad Sci U S A 1983;80:2534-2538. doi: 10.1073/pnas.80.9.2534.

[53] Guo Y, Li Y, Mu S, Zhang J, Yan Z. Evidence that methylation of hepatitis B virus covalently closed circular DNA in liver tissues of patients with chronic hepatitis B modulates HBV replication. J Med Virol 2009;81:1177-1183. doi: $10.1002 / j m v .21525$.

[54] Vivekanandan P, Thomas D, Torbenson M. Methylation regulates hepatitis B viral protein expression. J Infect Dis 2009;199:1286-1291. doi: 10. $1086 / 597614$

[55] Curradi M, Izzo A, Badaracco G, Landsberger N. Molecular mechanisms of gene silencing mediated by DNA methylation. Mol Cell Biol 2002;22:31573173. doi: $10.1128 / \mathrm{mcb} .22 .9 .3157-3173.2002$.

[56] Belloni L, Allweiss L, Guerrieri F, Pediconi N, Volz T, Pollicino T, et al. IFN- $\alpha$ inhibits HBV transcription and replication in cell culture and in humanized mice by targeting the epigenetic regulation of the nuclear cccDNA minichromosome. J Clin Invest 2012;122:529-537. doi: 10.1172/JCI58847.

[57] Zhang Y, Mao R, Yan R, Cai D, Zhang Y, Zhu H, et al. Transcription of hepatitis $\mathrm{B}$ virus covalently closed circular DNA is regulated by $\mathrm{CpG}$ methylation during chronic infection. PLoS One 2014;9:e110442. doi: 10.1371/journal.pone. 0110442.

[58] Vivekanandan P, Thomas D, Torbenson M. Hepatitis B viral DNA is methylated in liver tissues. J Viral Hepat 2008;15:103-107. doi: 10.1111/j.1365-2893. 2007.00905.x.

[59] Vivekanandan P, Kannangai R, Ray SC, Thomas DL, Torbenson M. Comprehensive genetic and epigenetic analysis of occult hepatitis $B$ from liver tissue samples. Clin Infect Dis 2008;46:1227-1236. doi: 10.1086/529437.

[60] Rivière L, Gerossier L, Ducroux A, Dion S, Deng Q, Michel ML, et al. HBx relieves chromatin-mediated transcriptional repression of hepatitis $B$ viral ccCDNA involving SETDB1 histone methyltransferase. J Hepatol 2015;63: 1093-1102. doi: 10.1016/j.jhep.2015.06.023.

[61] Chong CK, Cheng CYS, Tsoi SY], Huang FY, Liu F, Seto WK, et al. Role of hepatitis B core protein in HBV transcription and recruitment of histone acetyltransferases to cccDNA minichromosome. Antiviral Res 2017;144:1-7. doi: 10.1016/j.antiviral.2017.05.003.

[62] Zhang W, Chen J, Wu M, Zhang X, Zhang M, Yue L, et al. PRMT5 restricts hepatitis $B$ virus replication through epigenetic repression of covalently closed circular DNA transcription and interference with pregenomic RNA encapsidation. Hepatology 2017;66:398-415. doi: 10.1002/hep.29133. 
[63] Ren JH, Hu JL, Cheng ST, Yu HB, Wong VKW, Law BYK, et al. SIRT3 restricts hepatitis $\mathrm{B}$ virus transcription and replication through epigenetic regulation of covalently closed circular DNA involving suppressor of variegation 3-9 homolog 1 and SET domain containing $1 \mathrm{~A}$ histone methyltransferases. Hepatology 2018;68:1260-1276. doi: 10.1002/hep.29912.

[64] Qian G, Hu B, Zhou D, Xuan Y, Bai L, Duan C. NIRF, a novel ubiquitin ligase, inhibits hepatitis B virus replication through effect on HBV core protein and H3 histones. DNA Cell Biol 2015;34:327-332. doi: 10.1089/dna.2014.2714.

[65] Wei ZQ, Zhang YH, Ke CZ, Chen HX, Ren P, He YL, et al. Curcumin inhibits hepatitis $B$ virus infection by down-regulating cccDNA-bound histone acetylation. World J Gastroenterol 2017;23:6252-6260. doi: 10.3748/wjg.v23.i34.6252.
[66] Song $M$, Sun $Y$, Tian J, He W, Xu G, Jing $Z$, et al. Silencing retinoid $X$ receptor alpha expression enhances early-stage hepatitis $B$ virus infection in cell cultures. J Virol 2018;92:e01771-17. doi: 10.1128/JVI.01771-17.

[67] Koumbi L, Pollicino T, Raimondo G, Stampoulis D, Khakoo S, Karayiannis P. Hepatitis B virus basal core promoter mutations show lower replication fitness associated with ccCDNA acetylation status. Virus Res 2016;220: 150-160. doi: 10.1016/j.virusres.2016.04.022.

[68] Wu M, Li J, Yue L, Bai L, Li Y, Chen J, et al. Establishment of Cre-mediated HBV recombinant ccCDNA ( $r c c c D N A)$ cell line for cccDNA biology and antiviral screening assays. Antiviral Res 2018;152:45-52. doi: 10.1016/j. antiviral. 2018.02.007. 